\title{
Advancing the visualization of pure water transport in porous materials by fast, talbot interferometry-based multi-contrast $x$-ray micro-tomography
}

\author{
Fei Yang*a,b, Michele Griffa ${ }^{\mathrm{a}}$, Alexander Hipp ${ }^{\mathrm{c}}$, Hannelore Derluyn ${ }^{\mathrm{d}}$, Peter Moonen ${ }^{\mathrm{e}, \mathrm{f}}$, Rolf \\ Kaufmann ${ }^{\mathrm{a}}$, Matthieu N. Boone ${ }^{\mathrm{g}}$, Felix Beckmann ${ }^{\mathrm{c}}$, Pietro Lura ${ }^{\mathrm{a}, \mathrm{b}}$ \\ a Swiss Federal Laboratories for Materials Science and Technology (Empa), Überlandstrasse 129, \\ 8600 Dübendorf, Switzerland; ${ }^{b}$ Swiss Federal Institute of Technology in Zurich (ETH Zurich), \\ Stefano-Franscini-Platz 3, 8093 Zürich, Switzerland; ${ }^{c}$ Helmholtz-Zentrum Geesthacht, Max-Planck- \\ Strasse 1, 21502 Geesthacht, Germany; ${ }^{\mathrm{d}}$ UGCT/PProGRess, Dept. of Geology, Ghent University,

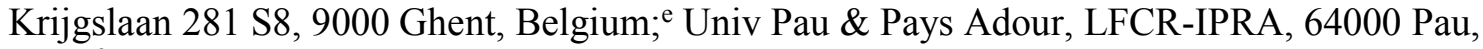 \\ France; ${ }^{\mathrm{f}}$ Univ Pau \& Pays Adour, DMEX-IPRA, 64000 Pau, France; ${ }^{\mathrm{g}}$ UGCT/Dept. of Physics and \\ Astronomy, Ghent University, Proeftuinstraat 86, 9000 Ghent, Belgium.
}

\begin{abstract}
The spatio-temporal distribution (4D) of water in porous materials plays a fundamental role in many natural and technological processes. The dynamics of water transport is strongly entangled with the material's pore-scale structure. Understanding their correlation requires imaging simultaneously the $4 \mathrm{D}$ water distribution and the porous microstructure. To date, 4D images with high temporal and spatial resolution have been mainly acquired by attenuation-based X-ray micro-tomography, whereby pure water is substituted by saline solutions with high atomic number components to improve image contrast. The use of saline solutions is however not always desirable, as the altered fluid properties may affect the transport process as well or, as it is the case for hydrating cement-based materials, they may modify the chemical reactions and their kinetics. In this study, we aimed at visualizing pure water transport in porous building materials by a new implementation of fast Talbot interferometry-based multi-contrast X-ray micro-tomography at the P07 beamline of the Helmholtz-Zentrum Geesthacht at DESY. We report results from a mortar specimen imaged at three different stages during evaporative drying. We show the possibility of visualizing simultaneously the microstructure and the pore-scale water redistribution by the phase contrast images. In addition, different solid material phases are clearly distinguished in these images. The higher contrast between water and the porous substrate, achievable in the phase contrast images, compared with the attenuation ones, empowers new analysis and allows investigating the correlation between the drying process and the porous microstructure. The approach offers the possibility of studying other chemically inert or reactive water transport processes without any chemical or physical perturbation of the processes themselves.
\end{abstract}

Keywords: porous materials, pure water transport, Talbot interferometry, X-ray phase contrast imaging, microtomography, porous microstructure

\section{INTRODUCTION}

Water transport occurs in several natural and man-made porous materials and is relevant for a large variety of natural and technological processes. For example, the transport of saline solutions in porous building materials contributes to their chemo-mechanical degradation ${ }^{1}$, and the loss of evaporative water leads to drying shrinkage and possibly to cracking, which, reduces the durability of porous building materials ${ }^{2}$. The spatial and temporal distribution of water thus plays a fundamental role in the materials' structure and overall macroscopic behavior. Conversely, the materials' pore-scale structural features, e.g., the pore space connectivity and the pore size distribution, largely influence the water transport process itself.

*fei.yang@empa.ch; phone 0041058765 4299; fax 0041058765 6935; empa.ch

Developments in X-Ray Tomography X, edited by Stuart R. Stock, Bert Müller, Ge Wang, Proc. of SPIE Vol. 9967, 99670L · (c) 2016 SPIE · CCC code: 0277-786X/16/\$18 · doi: 10.1117/12.2236221 
In this work, we focus on evaporative drying as one type of water transport process in porous materials. It is a complex process characterized by different stages where the local water content changes and the water spatio-temporal distribution are driven by different pore-scale processes in each drying stage. A significant amount of work has been dedicated to developing computational models of these pore-scale processes ${ }^{3}$ or to investigate them with experimental model systems, e.g., colloidal suspensions ${ }^{4}$. Time-resolved imaging of drying in real time in the actual porous materials of interest has been extensively pursued but, so far, with several limitations. Among all, Neutron Imaging (NI), Magnetic Resonance Imaging (MRI) and X-Ray Attenuation Contrast Imaging (XACI) have been the most frequently used, especially for porous building materials ${ }^{5,6,7}$.

NI achieves high image contrast to local water content, due to the large interaction cross-sections between neutrons and hydrogen. The temporal resolution of up-to-date neutron facilities is also sufficient to track water transport processes as capillary imbibition in porous materials. The main drawbacks of NI consist of the small number of neutron facilities available in the world and of the limited temporal access to them for each experiment. In addition, the achievable spatial resolution with the standard technology is currently limited to around $10 \mu \mathrm{m}^{8}$. New and future developments will push the limit to a few $\mu \mathrm{m}^{9}$.

MRI has the capability of quantitatively mapping the local water content and of estimating the pore size distribution at the same time (by Nuclear Magnetic Resonance relaxometry methods) with laboratory-scale setups. However, its temporal and spatial resolutions are even lower than what can be achieved by $\mathrm{NI}^{10}$.

X-ray imaging has been increasingly and widely used for studying liquid transport processes, including evaporative drying, in porous materials ${ }^{11}$. The standard contrast mechanism exploited consists of the X-ray attenuation and is quantified by the linear attenuation coefficient $\mu$, which relates with $\beta$, the imaginary part of the complex refractive index, $n=1-\delta+i \cdot \beta, \delta$ being the decrement of the real part. XACI has proven to provide sufficiently high spatial and temporal resolutions at both laboratory-scale ${ }^{12}$ and synchrotron radiation facilities ${ }^{13}$ to visualize pore scale water content change within materials with pores at the hundreds of $\mu \mathrm{m}$ to $\mathrm{mm}$-scale and/or with large water content changes ${ }^{14}$. However, for porous materials with pores at $\mu \mathrm{m}$ to nm-scale, this imaging approach does not provide enough image contrast to track the pore-scale pure water movement, because of (1) the large difference between the $\beta$ value of the solid matrix and that of water and (2) the voxel-wise small amount of water content change. In order to increase the attenuation contrast in XACI, pure water is therefore typically substituted with a water-based solution of salt containing atoms with high atomic numbers, e.g., $\mathrm{CaI}^{15}$.

The use of water-based salt solutions instead of pure water cannot be used with all water transport processes. First of all, the use of the solution changes the interfacial and fluid dynamic properties of the involved liquid, compared with water. Second, it leads to the increase of beam hardening artefacts ${ }^{16}$. Third and more importantly, it is unfeasible to use waterbased salt solutions instead of pure water when the transport process is chemically reactive, e.g., water transport through cement-based materials, due to the cement hydration reaction, or water transport in polymer electrolyte membrane fuel cells under operating conditions.

$\mathrm{X}$-ray phase contrast imaging (XPCI) techniques have shown their potential in visualizing pure water transport in porous materials with sufficiently high contrast ${ }^{17,18}$. In XPCI, the image is formed by retrieving phase shift information created by X-ray refraction and embedded in the transmitted beam intensity. Hence, it enables to map the spatial distribution of the decrement of the real part of the refractive index, $\delta$, above the imaging system's spatial resolution ${ }^{19,20}$.

For cement-based materials, as well as other porous materials, XPCI is preferable over XACI to visualize the pore-scale pure water transport for the following reasons: (1) the larger values of the ratio $\delta / \beta$ for water than for the material phases composing the porous substrate and (2) the smaller difference between the $\beta$ value of water and the $\beta$ value of air, normalized by the $\beta$ value of any solid phase of the porous substrate, compared with the corresponding normalized difference of $\delta$ values. The first feature indicates that, in a static specimen, pore-scale water content gradients may be more easily resolved in an image whose voxels are based upon $\delta$ values rather than upon $\beta$. The second feature suggests that any change in the specimen due to pore-scale water displacements will produce more significant voxel values changes in an image based upon $\delta$ than on $\beta$.

Talbot(-Lau) Interferometry (TI) is one of many XPCI techniques, and allows achieving multi-contrast X-ray imaging of pure water in porous materials with facilities both based on synchrotron radiation and laboratory-scale X-ray sources ${ }^{21}$. The image formation by TI is based on the changes brought by the insertion of the specimen within the X-ray beam when the beam has a periodic intensity distribution in the direction orthogonal to the propagation direction. Such 
periodic pattern, locally perturbed by the specimen, is an interference pattern due to the Talbot self-imaging effect ${ }^{22}$ generated when the X-rays illuminate a so-called phase grating, here called G1. Three distinct types of projection, based on contrast rising from different features inside the specimen, can be formed, namely an attenuation contrast projection image, a differential phase contrast projection, and a dark-field contrast projection. The three images are respectively a linear projection of $\mu$, the first order partial derivative of the linear projection in the direction perpendicular to the grating lines and the linear projection of $\xi$, the latter being the ultra-small angle scattering power ${ }^{23}$. X-ray dark-field imaging has been proven also useful in visualizing local water content changes in porous materials, especially when the majority of the pores have a size below the spatial resolution ${ }^{24,25}$.

Talbot Interferometry has been widely used in different fields such as the biomedical, chemical and materials sciences. Usually, the interferometers are operated at energy $<40 \mathrm{keV}$ for specimens containing low-Z number constituents ${ }^{26,27}$. However, for investigating materials or specimens with high X-ray attenuation, e.g., porous building materials, the energy should be higher in order to image a representative volume while still achieving enough photon statistics in reasonable time. Different approaches have been established to perform TI at energies higher than $40 \mathrm{keV}$, by means of fabricating gratings designed for higher energy ${ }^{28}$ or inclined grating geometry ${ }^{29,30}$. TI designed at high energy ( $\left.53 \mathrm{keV}\right)$ and based upon synchrotron radiation has been shown to be capable of imaging a static, $10 \mathrm{~mm}$-thick mortar sample with $\mu \mathrm{m}$ spatial resolution in previous study ${ }^{31}$.

In our work, we aimed at further exploiting XPCI with high temporal and spatial resolutions based on high-energy, synchrotron-based Talbot Interferometry-based multi-contrast X-ray micro-tomography (TI-MCXMT) and applying it for studying fast water transport in highly-absorbing porous building materials. We report results evaporative drying from a mortar specimen initially highly saturated with pure water. We performed TI measurements on the same specimen at three stages of water drying, without the need of any contrast agent. The measurements exploited the new implementation of TI-MCXMT at the P07 beamline of the Helmholtz Zentrum Geesthacht at DESY. Endowed by the high brilliance and spatial coherence of the synchrotron radiation beam and the high spatial resolution of a fast detector prototype at P07, we have been capable of reducing the tomographic acquisition time, by performing TI with a single phase-grating. In addition, thanks to the fabrication of TI phase gratings designed for the high energy range ( $>50 \mathrm{keV})$, the TI-MCXMT at P07 has allowed us visualizing the pore-scale water content changes in a highly absorbing porous building material such as a mortar via phase contrast X-ray images with $\mu \mathrm{m}$-scale spatial resolution, within an energy range where the standard attenuation-based contrast between pure water and the distinct material phases would be insufficient.

In Section 2 we describe the setup for TI-MCXMT at P07 and the overall configuration needed to perform in-situ evaporative drying experiments while acquiring the TI-MCXMT datasets. We also describe the 3D image processing and analysis steps for characterizing the spatial-temporal distribution of water at two of the three drying stages investigated. Section 3 reports the results of the imaging and image analysis, which clearly show the usefulness of XPCI by TIMCXMT for visualizing the pore-scale changes in local water content. In Section 4, we summarize the key advantages of using XPCI, as generated by TI with a single grating and a fast and high spatial resolution detector, for investigating water transport in porous materials, especially when the specimens are large enough (several mm in thickness) to require higher energies than those typically used for TI both with synchrotron radiation and laboratory sources.

Evaporative drying is studied in this work because it has a large influence on the durability of porous building materials as cement-based ones. However, the imaging and image analysis methodologies presented in this work are directly applicable for studying other water transport processes in other types of porous materials, especially those which are chemically reactive, thus requiring the use of pure water.

\section{MATERIALS AND METHODS}

\subsection{Materials and specimen preparation.}

A mortar specimen was cast according to the following mixing design: 0.51 water-to-cement ratio (w/c), by mass; $50 \%$ of sand (average size 0.3-0.4 mm and maximum $1 \mathrm{~mm}$ ), 19\% of cement (CEM I $52.5 \mathrm{~N}$, mass volumetric density of

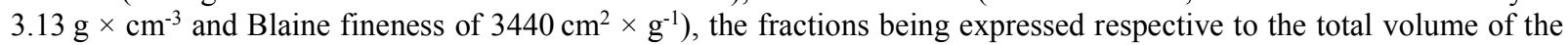
mix. After 91 days of curing, a $5 \mathrm{~mm} \times 19 \mathrm{~mm}$ (diameter $\times$ height) cylinder was cored out of the larger specimen with a diamond-based bore drill, while being flushed with water. After 5 days drying in the oven at $50{ }^{\circ} \mathrm{C}$, the sample was glued 
on a standard metallic specimen holder compatible with the P07 beamline. The lateral surface of the specimen was covered with a $70 \mu \mathrm{m}$ thick polyimide film, leaving only the top surface open, to drive the evaporative drying mainly along the symmetry axis of the specimen and the top surface being the only one available for water exchange with the environment. The specimen was initially saturated with de-ionized water under vacuum conditions (about 5 mbar) and kept in water till the $1^{\text {st }}$ tomographic scan.

\subsection{X-ray Talbot Interferometer at the P07 beamline, DESY, and evaporative drying/measurement protocols.}

The whole tomographic measurement campaign was carried out at the P07 beamline of the Helholtz Zentrum Geesthacht at DESY in Hamburg, Germany. We performed tomography on the same mortar specimen at three different stages: one before the start of the evaporative drying process, one at the beginning of the falling rate period and one at its end. At each stage, two regions of interest (ROIs) adjacent to each other along the vertical axis of the specimen were scanned. During each tomography, the specimen was located inside a small climatic chamber developed at Empa (Figure 1(A)), and flushed at a set flow rate with air at controlled temperature, and relative humidity (RH), produced by an air conditioner (GenRH-T ${ }^{\circledR}$ by Surface Measurement Systems Ltd). The actual RH and temperature in the chamber were measured by a Sensirion SH75 ${ }^{\circledR}$ sensor located just above the specimen, with data acquired by an in-house developed logging system based upon an Arduino $\mathrm{Nano}^{\circledR}$ micro-controller. The climatic chamber was necessary in order to reduce as much as possible any change in water content of the specimen during each tomography. The first tomographic scan, also called the reference scan, with the specimen still saturated with water, was performed at $80 \% \mathrm{RH}$ and $23^{\circ} \mathrm{C}$. The top of the sample was covered with a piece of polyimide tape further limit potential evaporative drying. After the first scan, the tape was removed and the specimen placed onto a high-precision $( \pm 0.1 \mathrm{mg})$ balance whose cabinet was sealed and contained silica gel particles, creating an inner atmosphere of $19.7 \%$ average $\mathrm{RH}$ and $23.7^{\circ} \mathrm{C}$ average temperature, driving the evaporative drying of the specimen. A sensor (ECOLOG TH1 ${ }^{\circledR}$ by ELPRO-BUCHS AG) was used to measure $\mathrm{RH}$ and temperature inside the cabinet of the balance. The first session of drying lasted 40 minutes, during which the specimen mass change was recorded automatically by the balance. The second tomographic scan followed the first drying session and was performed keeping the chamber at $45 \% \mathrm{RH}$ and $23{ }^{\circ} \mathrm{C}$, values chosen by repeated pre-tests in order to reduce as much as possible any re-wetting or continuation of drying of the specimen during the scan. The second scan lasted $2 \mathrm{~h} 50 \mathrm{~min}$ and during the scan, the top surface was again covered with polyimide tape to limit the drying. After the second scan, the specimen was moved back to the balance, kept at the same conditions as before, for a second drying session, $8 \mathrm{~h} 11 \mathrm{~min}$ long, then it was moved into a desiccator containing silica gel particles for a final, long-term drying, with an atmosphere of $1-3 \% \mathrm{RH}$ and $23{ }^{\circ} \mathrm{C}$ average values, measured continuously as well with another sensor similar to the one used inside the balance. The third and final tomographic scan was performed after $37 \mathrm{~h} 44 \mathrm{~min}$ of drying into the desiccator.

A 'twin' specimen, of the same type and size as the scanned one, was used to estimate the eventual additional drying caused by the radiation dose imparted during the tomography scans. The 'twin' specimen went through the same preparation and drying protocol as the scanned specimen, except for not being illuminated by the beam. During the scans, it was instead kept in a corner of the climatic chamber. Before and after each drying stage and scan, the mass of both the twin and the scanned specimens was measured.

The principles for the operation and image acquisition using an X-ray Talbot interferometer are explained in details in Ref. $^{32,33}$. The experiment was performed with a X-ray energy of $55 \mathrm{keV}$. This value was selected based upon the specifications of the Talbot interferometer and preliminary test results on similar dried specimens. In this work, as shown in Figure 1 (B), the high spatial resolution detector and the high flux of the X-ray beam (source brilliance $10^{18} \mathrm{ph} / \mathrm{s} / 0.1 \% \mathrm{bw} / \mathrm{mA}$ ), allowed using a single phase grating-based setup, avoiding using an additional analyzer grating (G2) to detect and resolve changes in the periodic interference pattern. Thus, the temporal resolution of the TI measurement for each radiograph acquisition could be significantly increased compared to typical time scales with an additional grating analyzer. The phase grating was fabricated by IMT (Institute for Microstructure Technology, Karlsruhe Institute of Technology (KIT)) using LIGA processing, with $10.4 \mu \mathrm{m}$ nickel height and a period of $10 \mu \mathrm{m}$. It is designed to generate a $\pi / 2$ phase-shift for the transmitted X-ray beam at $60 \mathrm{keV}$, creating an interference pattern with equal periodicity as the phase grating's. The distance between the phase grating and detector was $800 \mathrm{~mm}$. The specimen was mounted on an air bearing rotational stage (Aerotech), at the distance of $1258 \mathrm{~mm}$ to the detector. The detector is a new experimental CMOS camera from HZG/KIT, with $5120 \times 3840$ pixels (width $\times$ height) and physical pixel size of $(6.4 \mu \mathrm{m})^{2}$. The transmitted X-ray beam was converted into a visible light beam by a $100 \mu \mathrm{m} \mathrm{CdWO}$ scintillator screen and conveyed on an optical microscope for magnification and projection on the CMOS camera. In our case, the final image pixel size was $1.28 \mu \mathrm{m}$ after a $\times 5$ optical magnification, which leads to an effective field of view (FOV) of $6.5 \mathrm{~mm}$ 
$\times 2.3 \mathrm{~mm}$ (width $\times$ height), considering only the middle 1797 pixel rows corresponding to the central, more homogeneous region of the beam's cross-section. Each specimen projection was acquired by a phase stepping protocol consisting of 3 steps (4 images) over 1 grating period. The detector exposure time at each stage of the phase stepping protocol was $0.12 \mathrm{~s}$. For one tomographic acquisition, we took 901 projections over $360^{\circ}$ of specimen rotation. 5 dark current images were acquired, without the presence of the beam before each tomographic acquisition, in order to estimate the noise floor of the detector, and 2 reference (flat field) projections, in the absence of the specimen were acquired after each 25 specimen projections. The total duration for one tomographic scan was approximately $1 \mathrm{~h} 30 \mathrm{~min}$, including the time spent on the specimen motion. For each state of specimen (wet, drying stage 1, drying stage 2), we acquired data for 2 tomograms, each corresponding to a ROI. The tomogram for the second ROI was acquired by moving up the sample $2.2 \mathrm{~mm}$ vertically, leaving $0.1 \mathrm{~mm}$ specimen region overlapped in height. The total duration of the two scans at the first measurement stage, before starting the drying, was approximately $6 \mathrm{~h} 25 \mathrm{~min}$, including more than $2 \mathrm{~h}$ pause due to beam absence. The duration of the two tomographic scans at the $2^{\text {nd }}$ and $3^{\text {rd }}$ stages, after the $1^{\text {st }}$ and $2^{\text {nd }}$ drying periods, was about $2 \mathrm{~h} 54 \mathrm{~min}$.

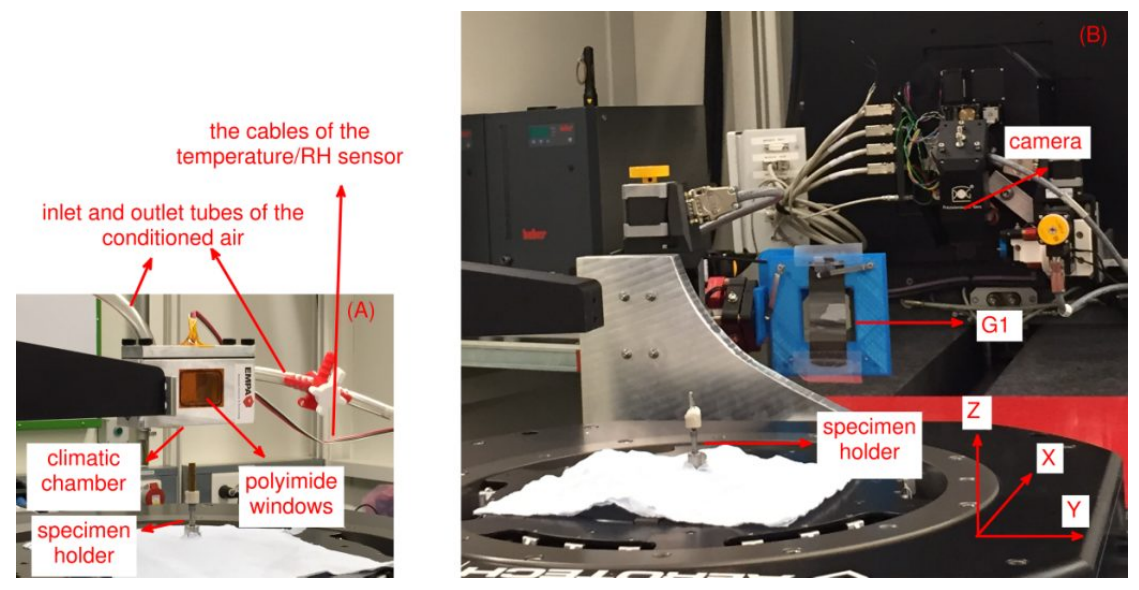

Figure 1. (A) The climatic chamber was connected to a motorized arm with three linear degrees of freedom. The vertical position of the chamber, along the $\mathrm{Z}$ axis indicated in inset (B), was decreased in order to bring the specimen inside the chamber through a small hole at its bottom. The chamber has two windows covered with a $50 \mu \mathrm{m}$-thick polyimide film, allowing the X-ray beam to be transmitted through the specimen with minimum attenuation and refraction while still guaranteeing good sealing of the chamber. The chamber has an inlet hole on the lateral surface and an outlet one on the top surface for the conditioned air to flow through it. A relative humidity and temperature sensor inserted into the chamber through its top surface allowed measuring those two variables exactly on top of the specimen. The air flow was set as such to achieve constant temperature and relative humidity conditions inside the chamber even though the bottom hole for the specimen insertion was not completely sealed. (B) The overall geometry and configuration of the Talbot interferometer at the $\mathrm{P} 07$ beamline. The optical axis (X-ray beam direction) was the positive $\mathrm{X}$-axis direction.

\subsection{Image retrieval, tomographic reconstruction and 3D image analysis.}

The attenuation and phase contrast image retrieval was performed using a Fourier analysis of the recorded stepping curve. For reconstruction of the volume the projections were binned by a factor of $2 \times 2$ before applying a filtered back projection algorithm with an imaginary kernel due to the differential data in case of the phase contrast images leading to a final voxel size of $2.56 \mu \mathrm{m}$ for the output reconstructed $3 \mathrm{D}$ images.

The 3D analysis was performed at Empa. In this paper, we focus only on the first and on the third scans of the top ROI. That ROI underwent the strongest water content changes between those two scans. Because the goal of this study was to exploit the high spatial and temporal resolutions of TL-MCXMT at P07 and the high contrast to water content changes achievable by XPCI, the X-ray attenuation contrast and dark-field contrast tomograms were not analyzed.

We performed 3D image registration on both XPCI tomograms with the commands Image $>$ Transform $>$ Translate and Image $>$ Transform $>$ Rotate in Image J/Fiji ${ }^{34}$, assuming only a combination of translation and rotation as the transformation mapping of one tomogram to the other. After registration, we selected 800 cross-sections in the X-Y plane. We will refer to such cross-sections as slices in the following paragraphs. 
We corrected for the voxel value offset between the two tomograms by subtracting voxel-wise from each of them the averaged voxel value calculated within an identical 3D ROI not containing the specimen volume. In order to reduce the influence of ring artefacts in the segmentation results, we applied a 3D anisotropic diffusion filter to each tomogram, using the Perona-Malik algorithm ${ }^{35}$ as implemented within the Anisotropic Diffusion Module of the software Avizo $3 \mathrm{D}^{\circledR}$ 9.1.1 (FEI Visualization Sciences Group). We set the diffusion stopping parameter of that algorithm by a direct inspection of the blurring of the images by evaluating the changes in average voxel values and standard deviation thereof calculated from ROIs representing different material phases inside the specimen. Finally, each slice was cropped to a size of $1970 \times 2007$ pixels.

Finally, we segmented the regions containing only the specimen volume by a constrained region growing algorithm implemented in the Empa Bundle of ImageJ Plugins for Image Analysis, EBIPIA (plugin 'Segment Phase 3d') 36 . This yields a 3D binary (8-bit) image, referred to as "specimen mask" in what follows.

\subsection{Pore segmentation.}

In this study, we focused on water transport through the pores above the spatial resolution, i.e., larger than about $5 \mu \mathrm{m}$, estimating the effective spatial resolution approximately as the double of the voxel size $(2 \times 2.56 \mu \mathrm{m})$. The segmentation of pore voxels was done separately for the first tomogram and the third one. For each of tomogram, we assumed the existence of 4 classes of voxels, which correspond to 4 types of material phases within the specimen: (1) empty pores, (2) (partially) saturated pores and unresolved highly porous regions inside the cement matrix, usually called porous patches $^{37}$, (3) the cement matrix an (4) the aggregates. Segmentation was performed using Otsu's N-thresholding algorithm $^{38}$ implemented as a $M A T L A B^{\mathrm{TM}}$ function $^{39}$. We considered the voxels belonging to the two classes with low voxel value (1) + (2) as pore voxels if they were classified as such on both tomograms. Accordingly, we generated a 3D binary tomogram called "pore mask" in what follows.

With the pore mask, we analyzed the pore size (cumulative-) distribution (PScD) by performing a 'continuous' PScD analysis $^{40}$, via an algorithm implemented in the plugin 'Pore size distribution' of the EBIPIA and with similar algorithm as the ones described in Müller et al. (2002) and Fierz et al. (2008) $)^{41,42}$.

\subsection{Segmentation of in-pore water re-distribution}

The segmentation of water displaced inside the resolved pore space due to the evaporative drying was done using the 3D time-differential phase contrast tomogram, called "Diff-XPCI", which was generated by voxel-wise subtraction of the third tomogram from the first one. We performed the segmentation of regions where an in-pore water content change occurred between the two tomograms, i.e., between the two corresponding stages of the drying process, by assuming the existence of 3 classes of water content change: pore voxels that gained water; those whose change is too small to be ascribed to a local water content change (i.e., a change close to the noise floor of the time-differential tomogram), and those with water loss. The three classes of time-differential voxels have value from low to high, respectively.

We did a preliminary segmentation by evaluating the noise level of the time-differential tomogram. 10 cubic ROIs were selected within regions belonging to the aggregates, which are supposed to undergo no water content change during the experiment. The averaged value of those selected voxels was calculated for the first tomogram, $P_{a}^{1}$, and Diff-XPCI, $\Delta P_{a}$. We defined a minimum threshold, called $\mathrm{TH}$, for considering a voxel value in Diff-XPCI as due to an in-pore water content change as:

$$
T H=\left|\Delta P_{a} / P_{a}^{1}\right|
$$

and classified each pore voxel of Diff-XPCI into three classes using the criteria listed below:

if $\Delta P_{\text {pore }}>0 A N D\left|\Delta P_{\text {pore }}\right|>\left|T H \times X P C I_{\text {pore }}^{1}\right| \rightarrow$ pores with water loss

if $\Delta P_{\text {pore }}<0 A N D\left|\Delta P_{\text {pore }}\right|>\left|T H \times X P C I_{\text {pore }}^{1}\right| \rightarrow$ pores with water gain

if $\left|\Delta P_{\text {pore }}\right|<\left|T H \times X P C I_{\text {Ipore }}^{1}\right| \rightarrow$ pores without confirmed water change

where $X P C I_{\text {pore }}^{1}$ is the value of the single pore voxel of the first tomogram and $\Delta P_{\text {pore }}$ is that of Diff-XPCI.

A second stage of the segmentation consisted of a refinement of those 3 classes, in order to reduce the influence of ring artefacts that remained in Diff-XPCI. The Otsu thresholding algorithm was applied to the pore voxel value histogram of 
Diff-XPCI, with the assumption of classifying them into 5 groups: pore voxels with water gain, pore voxels with nonconfirmed water change and 3 other classes with different water-loss levels. We restricted the pore voxels with water loss to those with values larger than $\operatorname{Max}\left(\Delta P_{\text {pore, } 2 \text { nd group }}\right)$, the latter being the maximum voxel value of the $2^{\text {nd }}$ group, based on the preliminary segmented water loss pore voxels. A 2-class Otsu thresholding was applied to the preliminarily segmented water gain pore voxels in Diff-XPCI. Out of the latter thresholding, we only considered the class with lower voxel value range, or in other words, with a significant change in voxel value between the 2 scans. During the whole process of refining the segmentation, we checked the validity of the selected thresholds by directly evaluating the voxel value of falsely segmented voxels due to ring artefacts and an interactive visual analysis of the final segmentation result.

\section{RESULTS AND DISCUSSION}

\subsection{Characterization of the evaporative drying experiment}

During the drying experiment, we measured the mass of the specimen in order to monitor the progress of the drying process. The mass evolution allows making assumptions about the temporal evolution of the water spatial distribution, based upon what is already known in the literature about evaporative drying of porous materials, especially from pore network computational modeling of the process. Such assumptions help in the interpretation of the image analysis results presented below about the actually observed spatial distribution of water content changes.

In Figure 2 (A) and (B), we plotted the recorded specimen mass, as well as the RH and the temperature of the specimen environment, at each step of the whole measurement campaign. The RH and temperature time series in Figure 2 (B) show the good conditioning of the specimen during drying within the balance cabinet and within the desiccator, at low relative humidity, intervals (2), (4) and (5). It can be observed that the relative humidity inside the balance cabinet was still dropping during the first drying stage, (2), while the other drying stages occurred at approximately constant and low $(\leq 5 \%) \mathrm{RH}$. Approximately constant or slow varying RH was also achieved inside the climatic chamber during first, at $80 \%$, interval (1), and the second scan 45\%, interval (3). Temperature and RH values are not reliable during the second scan, interval (3), or available during the third scan, interval (6), due to radiation damage of the sensor. As the settings of the air conditioner were the same as during interval (3), we expect similar values.

Table 1 lists the absolute mass change (loss) of the specimen during the 6 experimental steps indicated in Figure 2 (A).

Figure 2 (A) shows that the specimen lost a considerable amount of water due to the time needed for the measurement setup prior to the first scan and due to the $2 \mathrm{~h}$ beam absence during the first scan. A mass loss is observed also during the second scan, interval (3) in Figure 2 (A). The high RH inside the chamber did not allow achieving a perfect "freezing" of the drying process, thus the tomographic scans are expected to be affected by the changing water content.

Despite these experimental limitations, the mass loss during the scans was lower than the loss obtained during the drying inside the balance cabinet. A significant difference in the drying rate can be observed between the first, short drying period, interval (2), and the second drying period, interval (4): the first order time derivative of the mass time series significantly decreases from (2) to (4). The same can be inferred for the drying in the desiccator, interval (5).

Based upon these data, the picture we draw about the drying kinetics stages is the following.

The specimen was already within the so-called constant drying rate period (CRP) during the first scan and the first drying period, (1) + (2). One of the pore-scale mechanisms driving drying is the evaporation of water inside pores, starting from the top surface. Water menisci get formed, increasing the local capillary pressure. Larger pores get emptied first and more easily. While the thickness of material, experiencing the emptying of its pores (water loss), increases with time, with air substituting water (air invasion), a complicated network of smaller and interconnected pores gets refilled with water (water gain) due to the different capillary pressure values in pores with different sizes, corresponding to gradients in pore pressure. Such network can extend itself up to the top evaporation surface and can supply water to it. The macroscopic water loss occurs then through in-pore water evaporation and the following slow water vapor diffusion plus direct evaporation at the surface, with a constant supply of liquid water to it. The CRP is thus characterized by a complicated dynamics of emptying and filling of pores, with the larger pores mainly undergoing a loss of water.

Evaporation inside the network of smaller and re-filled pores leads at a certain moment to its breakage, with loss of percolation from the top surface to the bottom region still completely saturated. From that moment on, the supply of liquid water to the top surface is interrupted, pore pressure gradients mainly displace water locally, inside small clusters 
of smaller pores and vapor diffusion becomes the principal process driving the macroscopic water loss, thus proceeding at lower and decreasing speed. The drying enters into the so-called falling rate period (FRP).

The second drying stage in our experiment, (4), was characterized not only by a lower mass loss rate but also by a nonconstant one, compared with the first drying period, (2). We thus assume that the transition from the CRP to the FRP occurred either during (2) or (3), while the specimen was already in an advanced stage of the FRP during (4) and (5).

Thus, at the time of the third scan, the top ROI scanned should have been characterized by a multitude of small and disconnected clusters of pore regions either completely dried out or filled in with water due to localized pore pressure gradients. The saturation degree should have thus undergone a significant drop between the first and the third scan, especially within the scanned and analyzed top ROI.

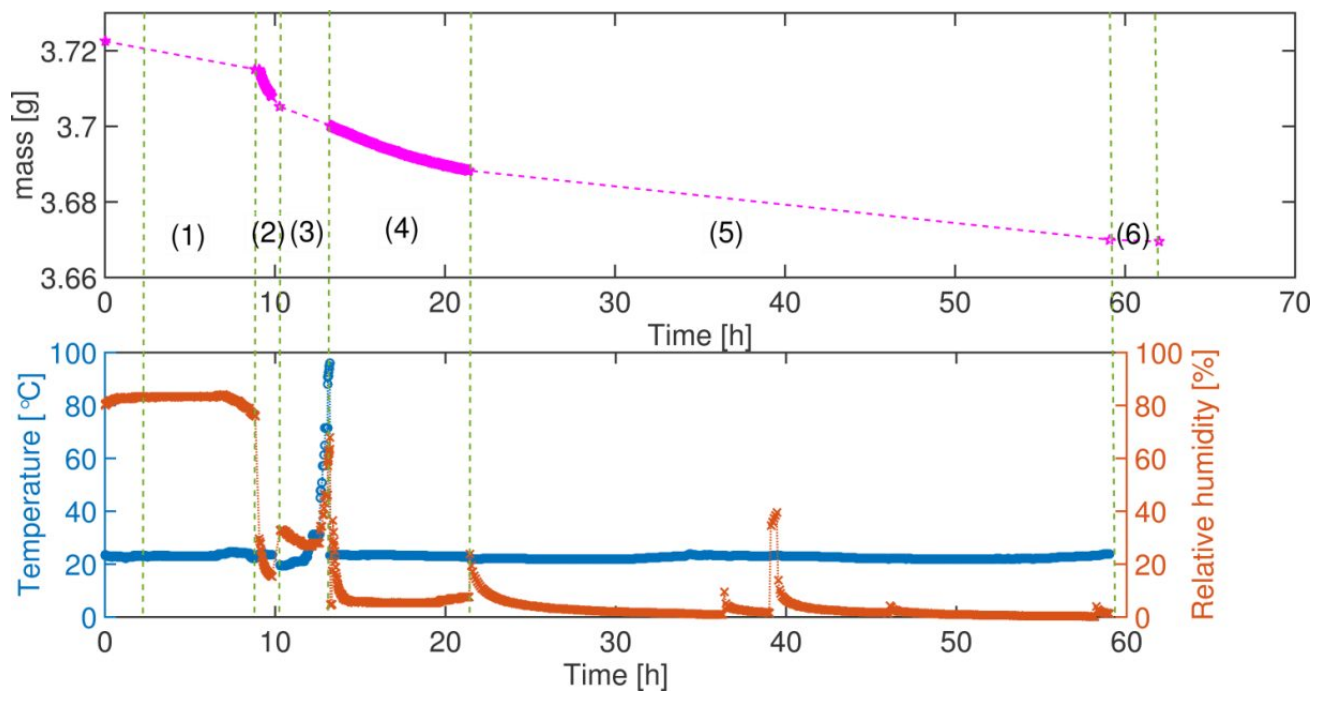

Figure 2 (A) Record of the specimen mass (purple, dashed, pentagram) throughout the measurement campaign. (B) Record of the temperature (blue, dotted, circle) and relative humidity (orange, dotted, cross) values of the environment where the specimen was stored during the experiment. Time 0 is the time when the specimen was moved into the experimental hutch. The whole time range is divided into 6 intervals, each delimited by two vertical dashed green lines and labeled with a number. (1): $1^{\text {st }}$ scan, before drying. (2): drying within balance cabinet, after the $1^{\text {st }} s c a n ;(3): 2^{\text {nd }}$ scan, after a short period of accelerated drying; (4): second drying stage within the balance cabinet, after the $2^{\text {nd }}$ scan; (5): long-term drying within the desiccator; (6) $3^{\text {rd }}$ scan, after the long-term drying. The time interval before (1) was spent on parameters optimization for the TI measurements, specimen alignment and a pause due to the beam loss. Only within the intervals (2) and (4), the mass of the specimen was continuously measured by the balance. For the other intervals, only the values at the beginning and at the end of each interval are available.

In addition to the mass measurements for the scanned specimen, we also measured the mass of the twin specimen at any step. For the tomographic scan steps, the mass was measured before and after each scan. The relative mass changes during these scans were approximately the same (within the limits given by the balance tolerance level of $0.1 \mathrm{mg}$ ) for both the scanned specimen and the twin one. For example, the relative mass change (loss) during the $3^{\text {rd }}$ scan was approximately $0.01 \%$ for both specimens. This result indicates no significant perturbation of the specimen's water content change by the irradiation.

Table 1. Absolute mass loss at each step of the experiment. The step numbers correspond to the labels in Figure 2(A).

\begin{tabular}{|l|c|c|c|c|c|c|}
\hline step & 1 & 2 & 3 & 4 & 5 & 6 \\
\hline mass loss [g] & 0.007 & 0.009 & 0.005 & 0.012 & 0.018 & 0.0005 \\
\hline
\end{tabular}




\subsection{Comparison between phase and attenuation contrast images.}

Figure 3 shows 4 slices taken at the same Z-position of the specimen, (A) and (B) from the XPCI tomograms, the first and third ones respectively, (C) and (D) from the corresponding XACI tomograms. For each image type, the minimum of the minima and the maximum of the maxima of the two tomograms' voxel values were calculated, considering only the voxels within the specimen mask. These values were used as bounds of the dynamic range for both images, rescaling linearly that voxel value range to the interval of integer numbers [0; 65535] (conversion from 32 bit float images to 16 bit unsigned integer ones). This type of conversion for each image of the same type ensures a visual comparison without any bias.

The visual comparisons between Figures 3 (A) vs (B) and (C) vs (D), respectively, show a larger voxel value drop in the XPCI images than in the XACI ones. The water loss by the pores above the spatial resolution is directly recognizable by comparing Figure 2 (A) to (B), an effect better highlighted in the respective small insets as zoom-in windows of a small ROI. Moreover, it is also clearly visible that the cement matrix becomes darker with drying. Such a reduction of voxel value is expected, since the replacement of water by air in pores leads to a drop in $\delta$, which is positively and linearly proportional to the voxel value change in the images. Cementitious materials have a pore system with a broad size distribution, from $\mathrm{nm}$ to over hundreds of $\mu \mathrm{m}$ up to a few $\mathrm{mm}$ for large quasi-spherical, entrapped air voids. Among all the pores, the capillary pores, which constitute the majority of the pore system and have sizes below the spatial resolution of these tomograms, are highly interconnected and significantly contribute to any water transport process. Hence, the reduction of the voxel value in the cement matrix reflects a loss of water in the smaller pores which are not directly visible in our tomograms. Apart from the features we focus on, related to drying, the high contrast between different material phases is also clearly seen in the XPCI tomograms. In contrast, one can barely observe any water content change from the XACI tomograms, neither in the pores above the spatial resolution nor in the cement matrix. In addition, except for the bright boundaries between different material phases, resulting from edge enhancement effects due to the high coherence of the X-ray beam and the long propagation distance between the specimen and the detector, the contrast between different solid material phases is much smaller in the XACI tomograms, compared with what achieved in the XPCI ones. Finally, the XACI images are also characterized by a much higher noise level.

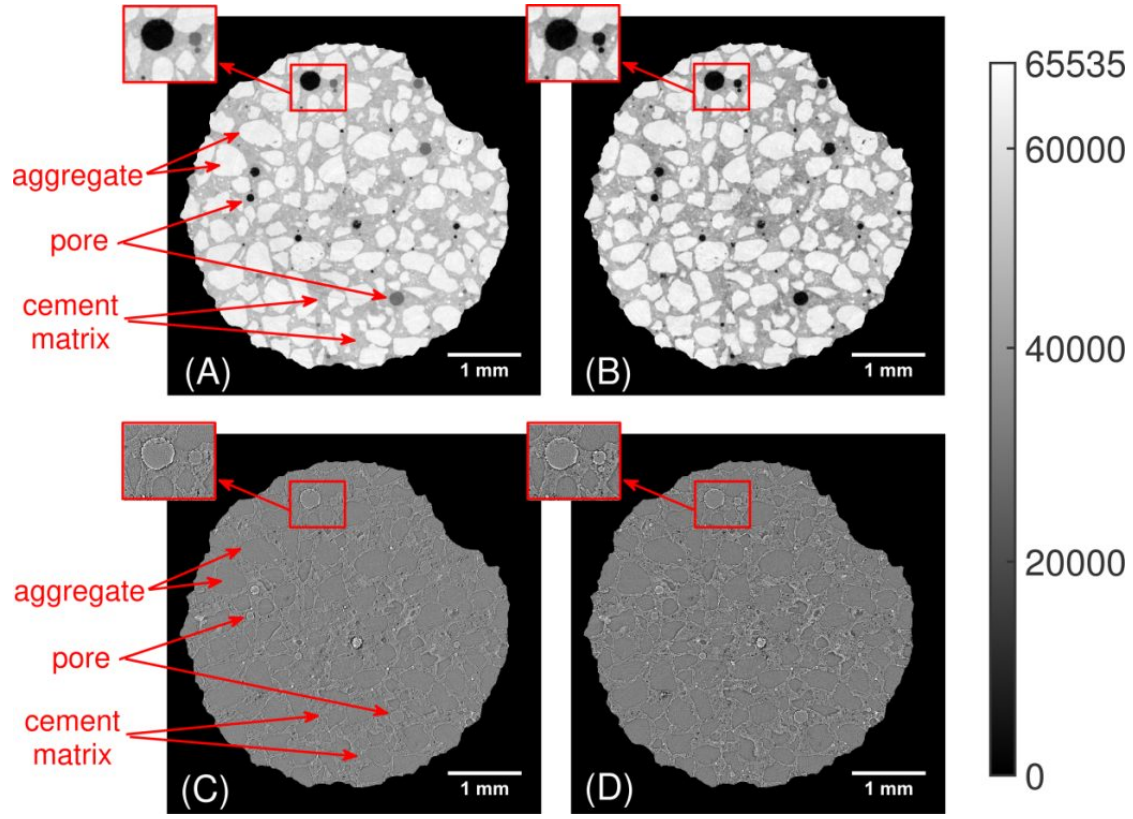

Figure 3. X-Y cross-section (slice) from the 3D X-ray phase-contrast, (A) and (B), and attenuation-contrast, (C) and (D), tomograms, respectively. Insets (A) and (C) correspond to the water saturated status of the sample at the $1^{\text {st }}$ scan, whereas (B) and (D) refer to the state of the specimen at the $3^{\text {rd }}$ scan, after the long term drying. Each tomogram of a given type of contrast was rescaled to unsigned 16 bit images, for unbiased visual comparison. Voxels belonging to the regions outside the specimen were assigned voxel value equal to 0 . Pores are darker while the solid material phases of the mortar are brighter. The brightest regions correspond to the aggregates, mainly made of $\mathrm{SiO}_{2}$. 
The reason for the higher contrast for local changes of water content in the XPCI tomograms than in the XACI ones is explained by Figure 4 , which show $\left(\beta_{\text {water }}-\beta_{\text {air }}\right) / \beta_{\text {solid }},\left(\delta_{\text {water }}-\delta_{\text {air }}\right) / \delta_{\text {solid }}$, and $\delta / \beta$ as a function of the X-ray energy $E$. The former two values represent the difference between the $\beta$ value of water and the $\beta$ value of air, normalized by the $\beta$ value for three types of solid phases of the porous substrate, and the corresponding normalized difference in $\delta$. $\beta$ is proportional to $E^{-m}$, with $m \cong 4-5$, and to $Z^{n}$, where $Z$ is the atomic number of the elements, with $n \cong 4-5$; $\delta$ is linearly proportional to $\mathrm{Z}$ and to $E^{-1}{ }^{43}$. The plots were generated using the NIST FFAST database of X-Ray Form Factor, Attenuation and Scattering Tables (FFAST) ${ }^{44}$. On the one hand, the value of $\delta / \beta$ for a single material achieves values as high as $10^{3}-10^{5}$ within the higher energy range including the $55 \mathrm{keV}$ of our measurements. Therefore, a XPCI tomogram, compared to a XACI one, shows an advantage in the detection of a water content change, because the change occurs with higher values. On the other hand, at the same energy level, $\left(\delta_{\text {water }}-\delta_{\text {air }}\right) / \delta_{\text {solid }}$ is approximately 15-20 times higher than $\left(\beta_{\text {water }}-\beta_{\text {air }}\right) / \beta_{\text {solid }}$ for the three main phases of the cementitious materials. If small amounts of water get displaced from one pore to another, the contribution brought by the displaced water to a pore region in the image is thus more detectable when the voxel value is based on $\delta$ than when it is based on $\beta$.

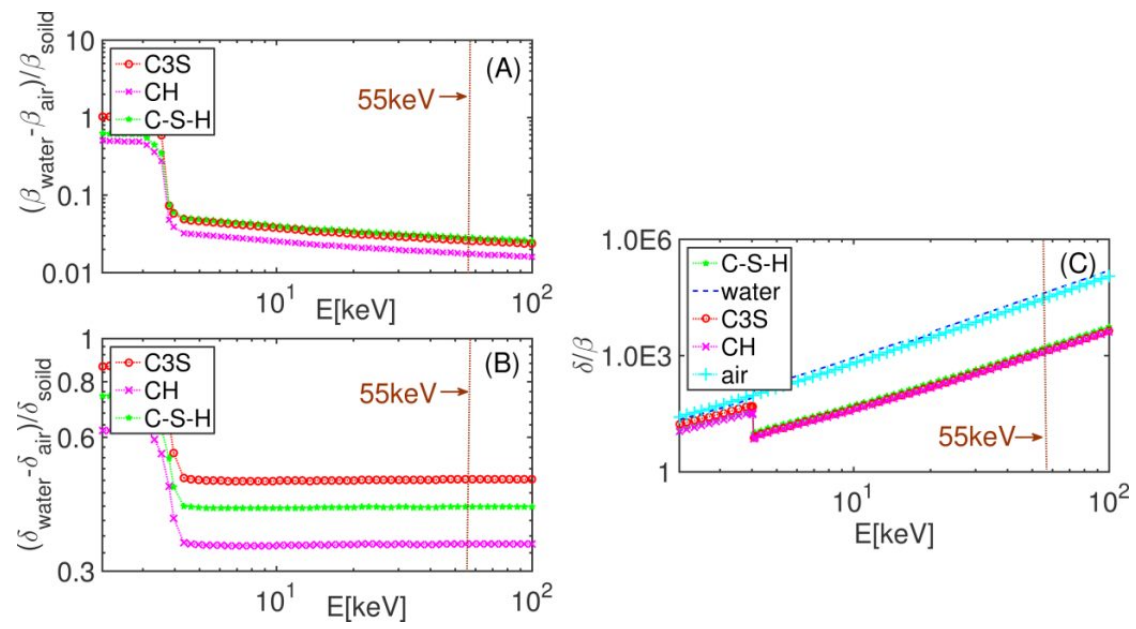

Figure 4. (A) Plot of $\left(\beta_{\text {water }}-\beta_{\text {air }}\right) / \beta_{\text {solid }}$, respectively the difference between the $\beta$ value of water and the $\beta$ value of air, normalized by $\beta$ value of the solid phase of the porous substrate; (B) the corresponding normalized difference in $\delta$, $\left(\delta_{\text {water }}-\delta_{\text {air }}\right) / \delta_{\text {solid }} ;(\mathrm{C})$ the ratio between real part and imaginary part of the complex index of refraction $n$, as a function of the photon energy $E$. The blue dashed line and the cyan dotted plus sign one in $(C)$ represent the values for water and air, respectively .The magenta dotted cross, red dotted circle and green dotted pentagram lines in (A), (B) and (C) correspond to the value of three main phases of cementitious materials, respectively, named according to the typical cement chemistry nomenclature system $\left(\mathrm{C}=\mathrm{CaO}, \mathrm{H}=\mathrm{H}_{2} \mathrm{O}, \mathrm{S}=\mathrm{SiO}_{2}\right)$ : $\mathrm{CH}$ (calcium hydroxide), $\mathrm{C}_{3} \mathrm{~S}$ (tri-calcium silicate), $\mathrm{C}-\mathrm{S}-\mathrm{H}$ (calcium silicate hydrate). The brown dotted lines in (A), (B) and (C) indicate the X-ray energy used in this study, which is $55 \mathrm{keV}$. The values for air are calculated as the value of gaseous nitrogen, $\mathrm{N}_{2}$, which contributes to up about $80 \%$ atmospheric air's composition.

\subsection{Voxel classes in the phase contrast images and segmentation of pore regions.}

The better visualization of different structural features and of the spatial water content change by XPCI is further shown by the XPCI voxel value histograms from the third and first scans in Figure 5 (A) and (B), respectively. The voxel value

shown in the histogram is the $\delta$ value, calculated by multiplying by a constant, $P / 4 \pi d$, the original voxel value obtained from the reconstruction, where $P$ is the period of the interference pattern, in our case, the period of the phase grating, and $d$ is the grating-to-detector distance.

Four voxel classes are better distinguished in the first XPCI tomogram, as shown in Figure 5(B), corresponding to different material features with voxel value from low to high: (1) empty pore voxels above the spatial resolution, (2) (partially-) saturated pore voxels above the spatial resolution and a small amount of voxels in the cement matrix region, containing unresolvable pores, (3) cement paste voxels and (4) voxels belonging to the aggregates. One could observe 
the strong reduction of voxels of the $2^{\text {nd }}$ class, as highlighted within the dashed green rectangle, Figure 5(A) and (B), and the corresponding increase of voxels of the $1^{\text {st }}$ class, as highlighted within the dashed red rectangles in both Figure 5(A) and (B), due to the emptying of resolvable pores after drying. The peak of the curve corresponding to the $3^{\text {rd }}$ class also broadens with drying, due to the loss of water in the cement paste, which has pores mostly at length scales below the spatial resolution of the tomograms.
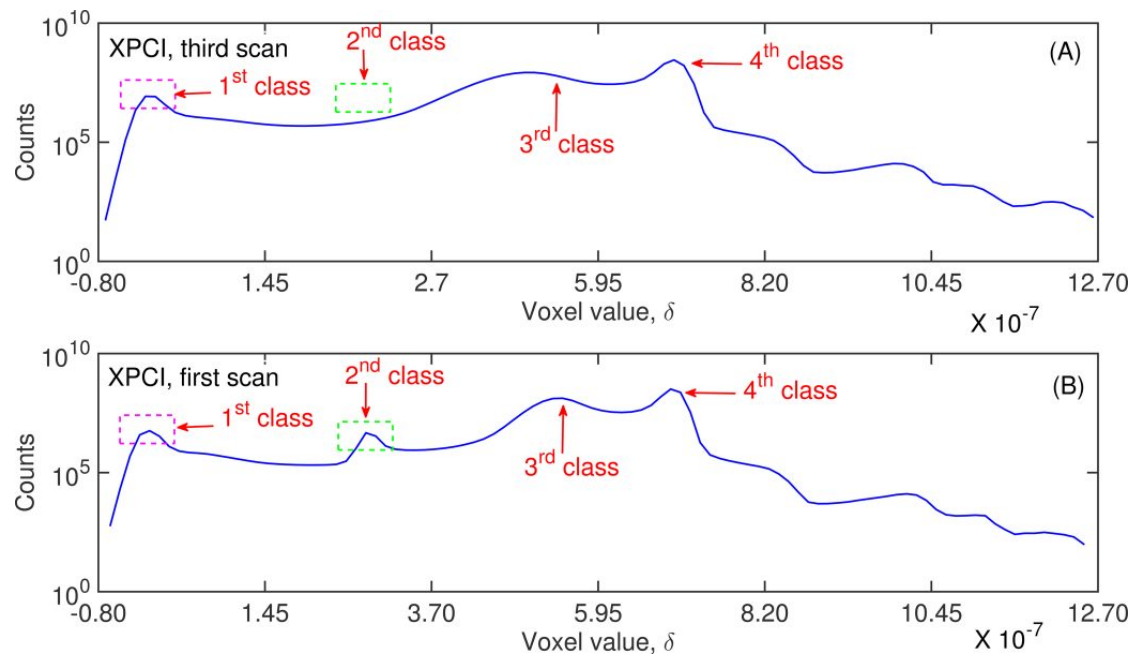

Figure 5. Voxel value distributions of the XPCI tomograms from the third scan, after the long term drying (A), and from the first scan, before the 1st drying stage (B). The voxel value shown is the $\delta$ value calculated by multiplying the original voxel value obtained by tomographic reconstruction with a constant. The value on the y-axis corresponds to the counts of voxels falling in each bin, reported with a logarithmic scale. The pink rectangles highlight the voxel class at the lowest value range, corresponding to the voxels of the empty or partially empty pores above the spatial resolution. The green rectangles in (A) and (B) highlight the class of voxels that belong to the saturated or partially saturated pores above the spatial resolution, which significantly decreased in number after drying. The 3rd class and 4th class of voxels refer to the cement paste voxels and the aggregate voxels.

Figure 6 provides one example of which voxels have been identified as pore voxels. The slice is taken at the same position of the specimen as the slices shown in Figure 3. The segmentation of the pore voxels and the pore size distribution analysis based on it indicates a total porosity of $2.3 \%$, considering only pores with sizes larger than the spatial resolution, i.e., about $5 \mu \mathrm{m}$. A 2-cycle mercury intrusion porosimetry (MIP) measurement was performed on additional specimens from the same batch of the specimen that was imaged to resolve the complete pore size distribution.

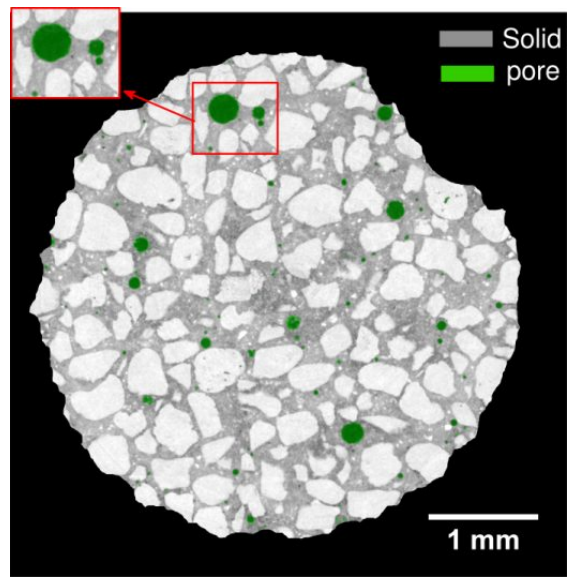


Figure 6. Overlapping of one slice of the $3^{\text {rd }}$ scan XPCI tomogram, after drying, and of the respective slice of the pore mask (semi-transparent green color). The small inset shows a zoom to a small ROI, as an example of the segmentation quality.

The slice is taken at the same position as that of Figure 3.

In Figure 7, we compare the cumulative pore size distribution curves obtained from MIP and from the 3D image analysis. The total porosity from experimental MIP is $23.39 \% .11 .29 \%$ porosity refers to ink-bottle pores ${ }^{45}$, which are more difficult to get emptied or re-filled during drying. The remaining $12.1 \%$ porosity refers to pores with more homogeneous geometry, thus easier to be dried up or re-filled. The latter pores could be responsible for the voxel value change within the cement matrix: even though not directly resolvable, these pores more likely participate in a more significant way to the drying process.

The presence of a strong ink-bottle effect is quite common for many porous building materials ${ }^{46}$. Due to this effect, the MIP measurements are characterized by a bias towards larger cumulative pore volumes at smaller pore sizes, i.e., the MIP curves tend to be shifted towards the left side of the pore size axis, compared to curves obtained by 3D image analysis. However, the 3D image analysis curve is limited by the spatial resolution, allowing estimating the pore size distribution only of a small fraction of the entire pore system, in this case $10 \%$ of the total pore volume.

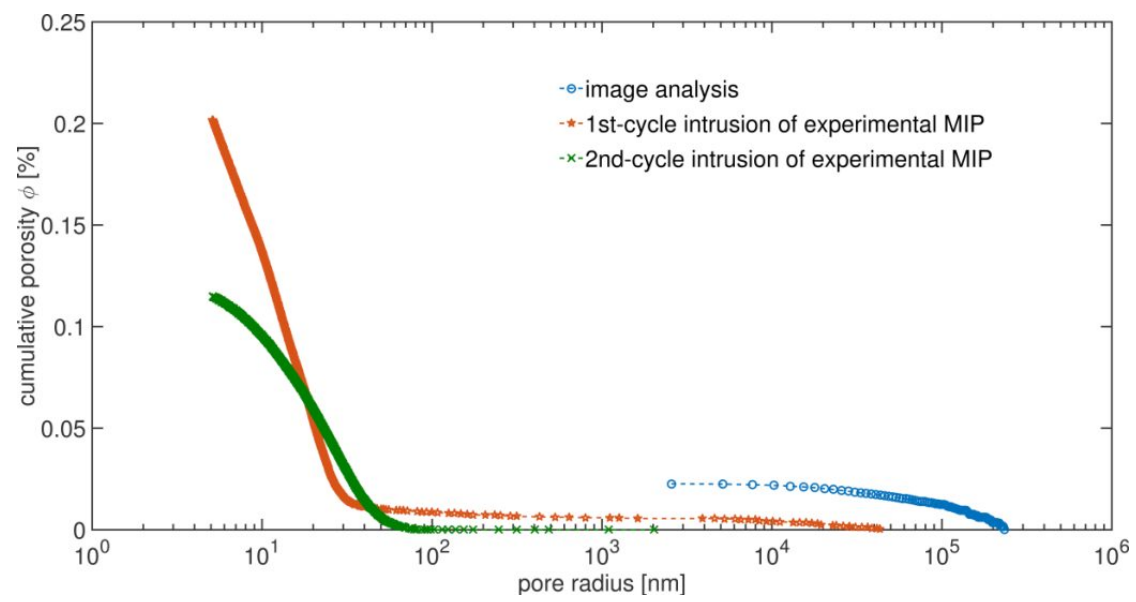

Figure 7. Pore size cumulative distribution (PScD) curves, obtained from the $1^{\text {st }}$-cycle intrusion (orange, dashed, pentagram) and from the $2^{\text {nd }}-$ cycle intrusion (green, dashed, cross) of experimental mercury intrusion porosimetry (MIP) and from 3D image analysis on the two XPCI tomograms (blue, dashed, circle). The cumulative porosity, as shown in vertical axis, is calculated by the cumulative pore volume divided by the sample volume.

\subsection{Time-differential phase-contrast images}

The spatial distribution of water content change due to drying between the $1^{\text {st }}$ and $3^{\text {rd }}$ scan can be better visualized by time-differential phase-contrast images. Figure 8 shows the slice taken at the same position as for Figure 3 but from the Diff-XPCI tomogram. For visualization purposes, the image has been rescaled to the dynamic range of [0;65535] according to the minimum and maximum values of the voxels belonging to the specimen mask. The voxels not belonging to the specimen region have been assigned value equal to 0 .

Brighter voxels in Figure 8 indicate a decrease in $\delta$, or in our case, a water loss, whilst darker voxels correspond to an increase in $\delta$, i.e., a water gain. The local increase of water content is due to water migration from larger to smaller pores, driven by a pore pressure gradients associated with different capillary pressure values for pores of different sizes.

Since no significant water content change is expected inside the aggregate regions, the average voxel value in those regions could be considered as a "0" level, with a certain variance due to image noise and remaining ring artefacts. The voxel value within the cement matrix is generally higher than within the aggregates. This might result from water displacement within and across unresolved pores. Comparing to the XPCI images in Figure 3, one can see that not all the pores have the same amount of water content change, or even some of them barely changed. 


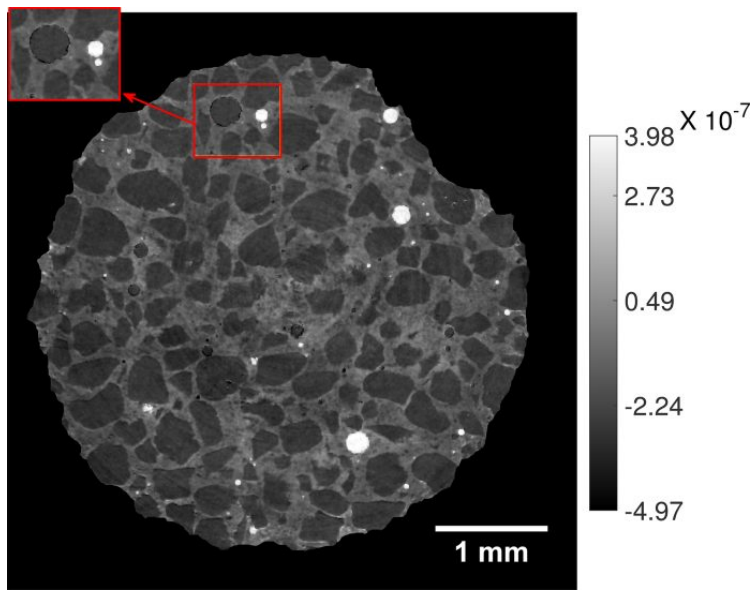

Figure 8. One slice from the 3D time-differential XPCI tomogram, calculated by voxel-wise subtraction of the tomogram after drying (third scan) from the tomogram before the $1^{\text {st }}$ drying stage (first scan). The original voxel value range was remapped by multiplying with a constant, which results in the measured $\delta$ value difference, as shown by the colorbar on the right. Brighter voxels indicate a local decrease in $\delta$, due to water loss, whilst darker voxels point to an increase, due to water gain.

The histogram in Figure 9 provides more quantitative information about the distribution of time-differential values for the voxels within the pore mask. Two main peaks are found to be centered within a lower and a higher voxel value range, respectively. The first range contains voxels without water content change or with water gain. The second range refers to voxels that lost a significant amount of water. The voxels with value distributed between the two peaks are considered as the ones with different degrees of water loss. There are also additional voxels with grey value being influenced by the ring artefacts, and we could neither clearly differentiate them from voxels characterized by a local water content change nor consider them with certainty as affected by any water content change.

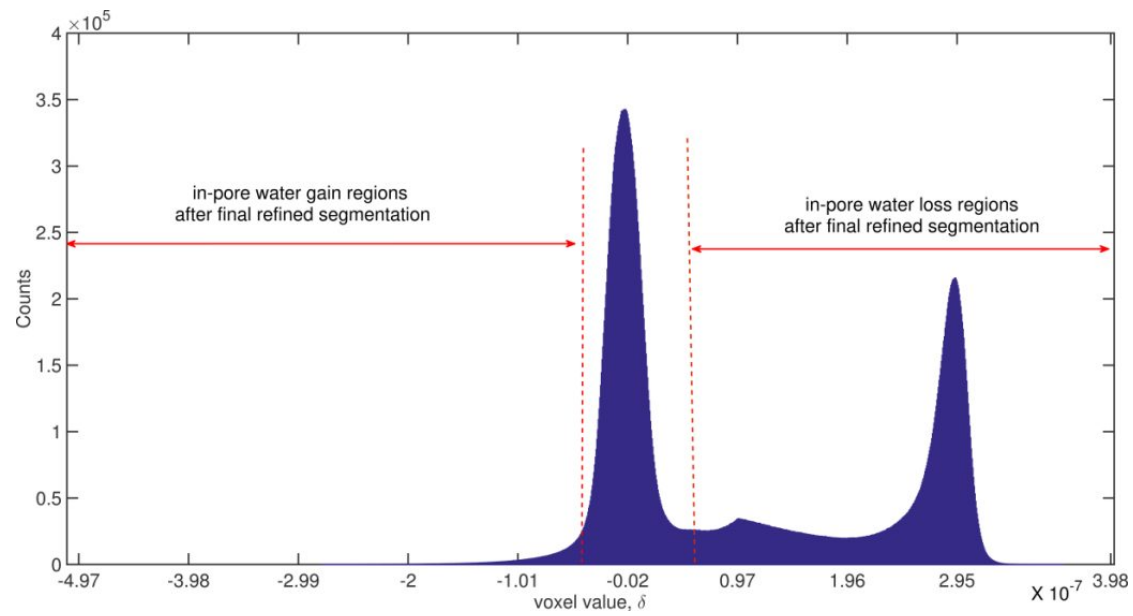

Figure 9. Voxel value histogram for the time-differential phase contrast tomogram, considering only the segmented pore voxels within the specimen volume. The voxel value shown in the plot represents the difference of measured $\delta$ values, being calculated by the original voxel value multiplied with a constant. The two voxel value intervals signed with double arrows at the left and right side of the histogram correspond to the final refined segmentation results of the regions that have significant water gain (left side) and loss (right side). The value range between these two intervals contains voxels without water content change or with too small change to be distinguished from noise and artefacts. 


\subsection{Classification of water content change regions and correlation with microstructural features}

Figure 10 provides two examples of segmentation results, visualized in 2D and 3D. It is shown that not all the resolved pores have lost or gained water between the first and the third scan (see the voxels color-coded in green in Figure 10 (A)). Possible reasons for the existence to these pores without water content change are: (1) these pores were empty already before the $1^{\text {st }} \operatorname{scan}$; (2) water filling/emptying occurred during the period between the two scans but before the third scan was performed these pores got emptied/re-filled again, due to local gradients in pore pressure; (3) these are pores with very narrow interconnection to other pores, so narrow to hinder the displacement of water or the water vapor diffusion through them, thus hindering the emptying of the pores themselves.

A small amount of the resolved pore space has gained water instead of losing it, which plays a key role in the capillaritydriven hydraulic transport processes besides in-pore water loss. Water loss pore regions are mainly distributed among the small resolved pores or are located at the boundary of larger pores. Small pores, especially when unsaturated at the beginning of the experiment, are more likely to receive water from larger ones due to capillary pressure gradients. The presence of water loss regions at the boundaries of large air voids, which have mostly lost water, could be explained by considering the possibility of the existence of very small pores, most of which likely close to or below the spatial resolution of the scan, connected to the air voids and representing some sort of neck pores for the air voids.
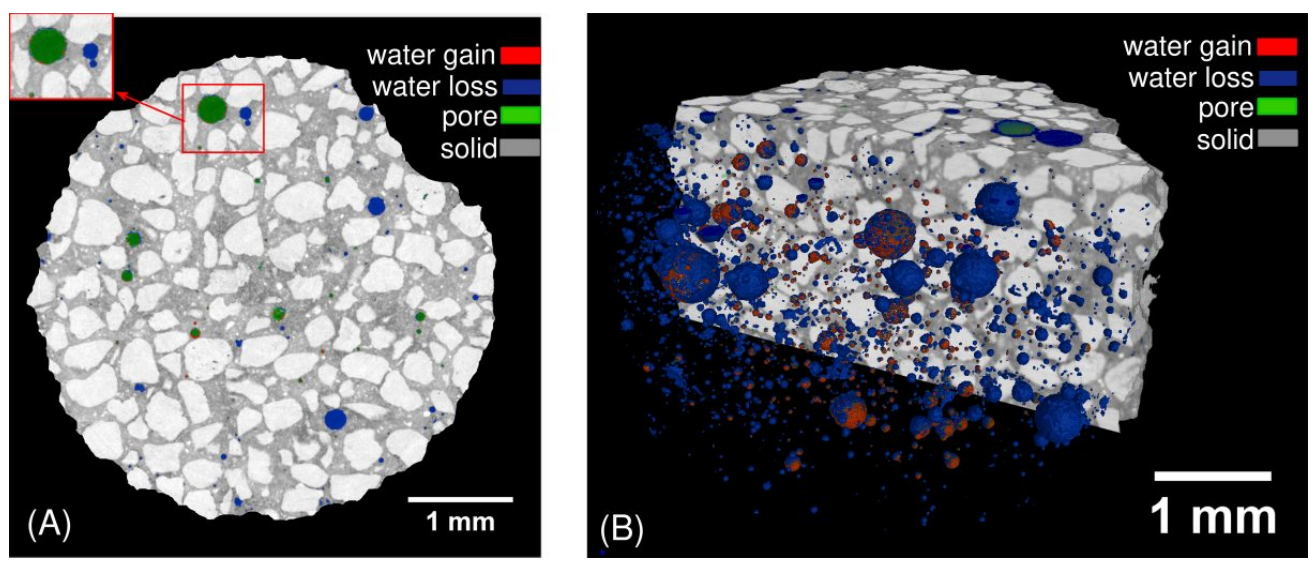

Figure 10 (A) Overlapping of one slice, at the same position as that in Figure 3, from the thrid scan tomogram, after drying, and of the respective slice from the pore mask (semi-transparent green color), the water loss mask (semi-transparent blue color) and the water gain mask (semi-transparent red color). Pores that are visible as green were classified as neither water loss nor water gain regions. The small inset shows a zoom into a small ROI, as an example of the segmentation results quality. (B) Volumetric rendering of the same 3D images.

A more quantitative analysis of the spatial distribution of water loss and water gain regions is provided by the plots in Figure 11. Here we calculated the water desaturation degree, shortly called DS. DS is defined as the ratio between the number of water-loss pore voxels and the total number of pore voxels within a given volume. We plotted DS as a function of the distance from the top of the analyzed ROI, Figure 11(A), choosing as calculation volume single slices. Similarly, we calculated the water saturation degree, S, defined as the ratio between the number of water-gain pore voxels and the total number of pore voxels. Figure 11(B) shows its linear profile along the depth from the top of the ROI, close to the specimen's evaporation surface, similarly to what is plotted in Figure 11(A) for DS.

The results show that in-pore water gain regions are uniformly distributed along the depth, as it should be expected, since water gain occurs by very localized displacements of water from larger to smaller pores due to pore pressure gradients which are spatially highly localized, but randomly distributed, especially during the falling rate period of the drying process, which is the drying period in which the specimen was at the time of the third scan, as explained in Section 3.1.

On the contrary, the desaturation degree shows a gradient with depth, with higher values close to the top of the ROI of interest, as it should be expected, since the larger pores loose on average more water the closer they are to the evaporation surface, especially during the constant rate period.

The total desaturation degree for the entire ROI analyzed was $46 \%$ while the saturation degree was $2 \%$. The remaining $52 \%$ of resolved pores was classified by our image analysis workflow as pores with no water content change. The 
specimen was very likely at an advanced stage of the falling rate period, as described earlier, thus one could expect a much higher desaturation degree. The difference between our image analysis results and what one could expect stem from the following two features.

First, we could segment only $10 \%$ of the total pore space, as evidenced by the MIP measurements (Figure 7). Thus, a part of the pore space with a strong role in the drying process is not considered in our analysis. Secondly, the pronounced ink-bottle effect shown by the MIP measurements (second intrusion curve in Figure 7), thus the large amount of pores accessible only through much narrower interconnecting pores, indicates a lower likelihood of drying for a significant amount of the larger pores, even during the constant rate period. The possibility of the large quasi-spherical air voids to be either disconnected from other parts of the pore space or to be connected only via very narrow neck pores with low degree of interconnection to other pores of similar size implies the existence of very large pore pressure gradients that could draw the water from the air voids but at the same time a lower likelihood that the water could be displaced at long distances towards the top of the specimen, hindering the drying rate, firstly during the constant rate period, thus also later on during the falling rate period.

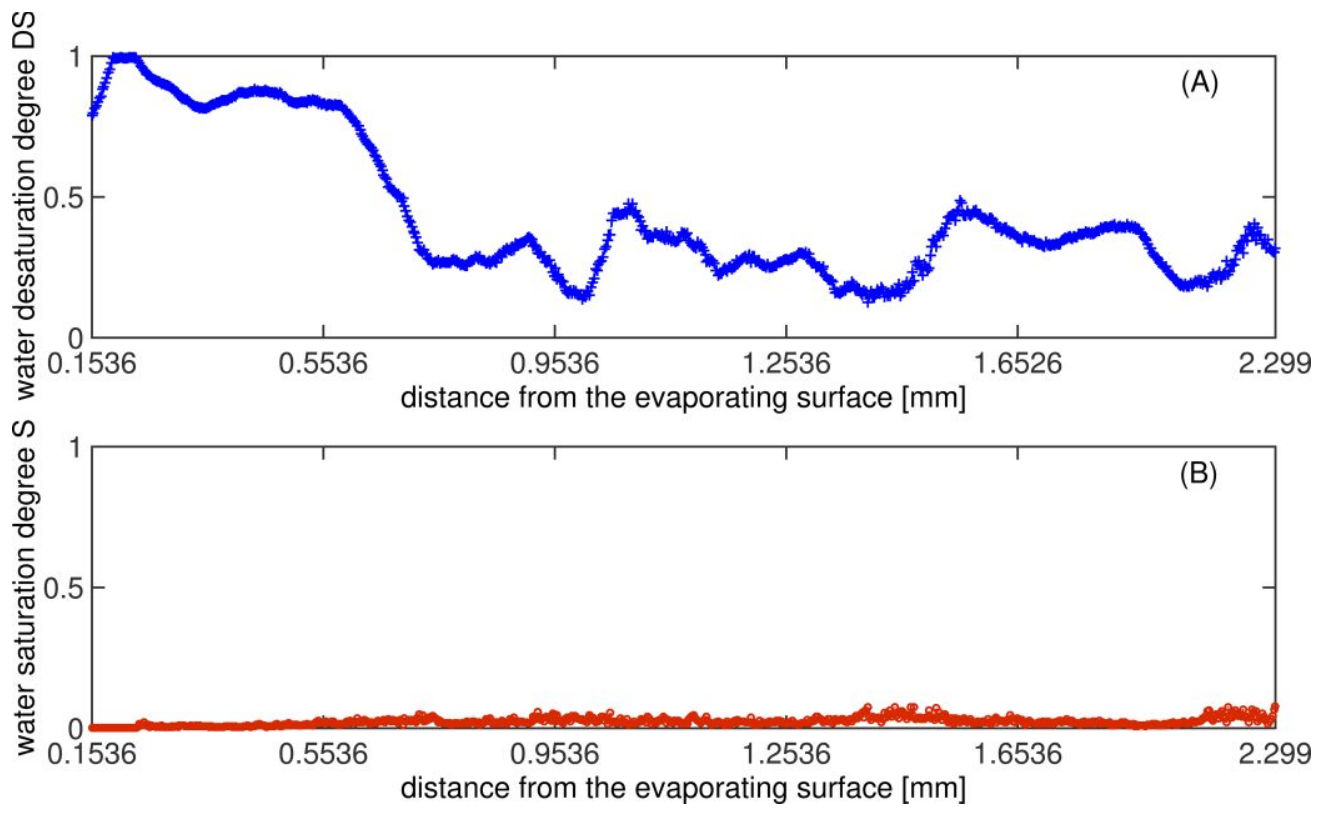

Figure 11. (A) Water desaturation degree, DS, as a function of the vertical distance from the evaporative surface; (B) water saturation degree, $\mathrm{S}$, as a function of vertical distance from the evaporative surface. The top of the analyzed sample ROI is about $0.15 \mathrm{~mm}$ to the real evaporating surface of the whole sample.

\section{SUMMARY AND OUTLOOK}

In this study we have performed multi-contrast X-ray micro-tomography with a synchrotron radiation-based, fast Talbot Interferometer on a mortar specimen while running an in-situ evaporative drying experiment. We show the capability of visualizing pore-scale water content changes without the need of "doping" the water with substances to increase its Xray attenuation, by exploiting the phase contrast instead of the attenuation mechanism. X-ray phase contrast imaging provides high contrast to local changes in content of pure water as well to different solid material phases. The key novelties of this in-situ experiment + imaging work consists of having been able to perform multi-contrast X-ray microtomography with high spatial and temporal resolution at higher energies than those typically used for the same type of multi-contrast X-ray micro-tomography, with Talbot interferometry, at other synchrotron radiation beamlines, allowing us measuring on a larger specimen without decreasing the temporal resolution of the tomographic imaging.

The 3D image analysis of the phase contrast tomograms shows the advantage of X-ray phase contrast imaging in studying at the pore scale water transport and its correlations with the specimen microstructure. In the specific case of the evaporative drying studied here, we were able to identify not only pore space regions affected by loss of water but also 
others which gain water, a phenomenon known to occur during drying because of very localized gradients in pore pressure due to formation of menisci in pores with different sizes.

The 3D image analysis of the second ROI inspected during our measurements along with the second scan tomograms (interval 3 in Figure 2), will allow us achieving further insight into the evolution of the spatial distribution of pore-scale water content changes not only at an advanced stage during the falling rate period of drying but also closer to its beginning, thus closer to the transition from the constant rate period. A multi- scale study of the full pore space of the specimen, e.g., combining these X-ray micro-tomography data with focused ion beam + scanning electron microscopybased nano-tomography to resolve smaller pores could help confirming our hypothesis about the role played by neck pores connected to larger air voids in hindering their water emptying, even during the constant rate period, leading to a better understanding of drying in cement-based materials.

Even though in our study we focused only on evaporative drying, our results suggest that the same experimental approach could be used for studying faster water transport processes, e.g., drainage or capillary imbibition, in porous materials, without the usage of any contrast agent, thus without any chemical and physical perturbation of the process itself, which is a sine qua non condition for studying reactive water transport processes as occurring in cement-based materials and in polyelectrolyte membrane fuel cells. The necessary further increase in temporal resolution for the Talbot interferometry-based micro-tomography could be achieved by, yet not limited to: 1) adopting experimental acquisition and image processing schemes which allow avoiding the phase-stepping protocol, e.g., the single-shot method ${ }^{47}$, already tested at the $\mathrm{P} 07$ beamline ${ }^{48}$ 2) improve the sensitivity of the scintillator, particularly the efficiency for the high-energy $\mathrm{X}$-ray photons 3 ) in the study of this manuscript, we performed the tomographic measurements by firstly rotating the sample, then completing the phase-stepping and repeating this procedure; in the future, the acquisition time could be reduced by firstly making one phase step, then rotating the sample over $180^{\circ}$ or $360^{\circ}$ and repeating this new procedure.

\section{ACKNOWLEDGEMENTS}

This work was performed under the framework of the Helmholtz Virtual Institute for New X-ray analytic Methods in Materials Science (VI-NXMM). We acknowledge the financial support of the Swiss National Science Foundation (project numbers 143782 and 162572), of the UGent BOF research fund $01 \mathrm{~B} 00512$ and the research grant $1521815 \mathrm{~N}$ of the Research Foundation - Flanders $(F W O)$, beyond that of the VI-NXMM. Hannelore Derluyn acknowledges the financial support from the FWO for her postdoctoral fellowship. The UGent Special Research Fund (BOF) is acknowledged for the post-doctoral grant of M.N.Boone. We would like to thank Roger Vonbank (Empa) and Dr. Marijn Boone (Ghent University) for guidance in the preparation of the Arduino-based data logging system for the temperature and relative humidity measurements inside the mini climatic chamber, Dr. Julia Herzen (HZG, now at Technical University Munich, TUM) for supervising Fei Yang during her exchange in HZG and persistently helping in the collaboration between Empa and HZG/TUM, Carmelo Di Bella (Empa) for providing us the mortar specimens along with respective MIP characterizations, Dr. Beat Münch (Empa) for inspiring discussions about efficient image processing/analysis methods and implementation of the PScD algorithm as a plugin in ImageJ and Erich Heiniger (Empa) and the overall Empa Wekstatt for the design and realization of the mini climatic chamber. Finally, we would like to thank all the HZG colleagues at the DESY station for all the support provided.

\section{REFERENCES}

[1] Derluyn, H., Griffa, M., Mannes, D., Jerjen, I., Dewanckele, J., Vontobel, P., Sheppard, A., Derome, D., Cnudde, V., Lehmann, E., and Carmeliet, J., "Characterizing saline uptake and salt distributions in porous limestone with neutron radiography and X-ray micro-tomography," J. Build. Phys. , 36(4) , 353-374 (2013).

[2] Scherer, G. W., "Drying, Shrinkage, and Cracking of Cementitious Materials," Transp. Porous Media. 110(2), 311-331 (2015).

[3] Prat, M., "Recent advances in pore-scale models for drying of porous media," Chem. Eng. J. 86, 153-164 (2002).

[4] Xu, L., Davies, S., Schofield, A. B., and Weitz, D. A., "Dynamics of drying in 3D porous media," Phys. Rev. Lett. 101(9), 29-32 (2008).

[5] Wyrzykowski, M., Trtik, P., Münch, B.,Weiss, J., Vontobel, P., and Lura, P., "Plastic shrinkage of mortars with shrinkage reducing admixture and lightweight aggregates studied by neutron tomography," Cem. Concr. Res. 73, 238-245(2015).

[6] Faure, P. F., Caré, S., Magat, J., and Chaussadent, T., "Drying effect on cement paste porosity at early age observed by NMR methods," Constr. Build. Mater., 29, 496-503(2012).

[7] Boone, M. A., De Kock, T., Bultreys, T. , De Schutter, G., Vontobel, P., Van Hoorebeke, L. and Cnudde, V., "3D mapping of water in oolithic limestone at atmospheric and vacuum saturation using X-ray micro-CT differential imaging," Mater. 
Charact., 97, 150-160(2014).

[8] Perfect, E., Cheng, C. L., Kang, M., Bilheux, H. Z., Lamanna, J. M., Gragg, M. J., and Wright, D. M., "Neutron imaging of hydrogen-rich fluids in geomaterials and engineered porous media: A review," Earth-Science Rev. 129, 120-135 (2014).

[9] Trtik, P., Hovind J., Grünzweig, C., Bollhalder, A., Thominet, V., David, C., Kaestner, A., and Lehmann, E. H., "Improving the Spatial Resolution of Neutron Imaging at Paul Scherrer Institut - The Neutron Microscope Project," Phys. Procedia. 69, 169-176 (2015).

[10] Mitchell, J., Chandrasekera, T. C., Holland, D. J., Gladden, L. F., and Fordham, E. J., "Magnetic resonance imaging in laboratory petrophysical core analysis," Phys. Rep. 526(3), 165-225 (2013).

[11] Wildenschild, D. and Sheppard, A. P., "X-ray imaging and analysis techniques for quantifying pore-scale structure and processes in subsurface porous medium systems," Adv. Water Resour. (51), 217-246 (2013).

[12] Bultreys, T., Boone, M. A., Boone, M. N., Schryver, T. De, Masschaele, B., Van Hoorebeke, L., and Cnudde, V., "Fast laboratory-based micro-computed tomography for pore-scale research: Illustrative experiments and perspectives on the future," Adv. Water Resour. 78, 36-49 (2015).

[13] Berg, S., Ott, H., Klapp, S. A., Schwing, A., Neiteler, R., Brussee, N., Makurat, A., Leu, L., Enzmann, F., Schwarz J.-O., Kersten M., Irvine S., and Stampanoni M., "Real-time 3D imaging of Haines jumps in porous media flow.," Proc. Natl. Acad. Sci. U. S. A., 110 (10), 3755-3759 (2013).

[14] Ketcham, R. A. and Iturrino, G. J., "Nondestructive high-resolution visualization and measurement of anisotropic effective porosity in complex lithologies using high-resolution X-ray computed tomography, " J. Hydrol. 302, 92-106 (2005).

[15] Shokri, N. and Sahimi, M., "Structure of drying fronts in three-dimensional porous media," Phys. Rev. E. 85 (6), 066312 (2012).

[16] Nakashima, Y., "The use of sodium polytungstate as an X-ray contrast agent to reduce the beam hardening artifact in hydrological laboratory experiments," J. Hydrol. Hydromechanics. 61(4), 347-352 (2013).

[17] Lee, J. S., Weon, B. M., and Je, J. H., "X-ray phase-contrast imaging of dynamics of complex fluids," J. Phys. D. Appl. Phys.46(49), 494006 (2013).

[18] Yang, F., Griffa, M., Bonnin, A., Mokso, R., Di Bella, C., Münch, B., Kaufmann, R., and Lura, P., "Visualization of water drying in porous materials by X-ray phase contrast imaging," J. Microsc. 261(1), 88-104 (2015).

[19] Gureyev, T. E., Mayo, S. C., Myers, D. E., Nesterets, Y., Paganin, D. M., Pogany, A., Stevenson, A. W., and Wilkins, S. W., "Refracting Röntgen's rays: Propagation-based x-ray phase contrast for biomedical imaging," J. Appl. Phys. 105(10), 102005 (2009).

[20] Diemoz, P. C., Bravin, A., and Coan, P., "Theoretical comparison of three X-ray phase-contrast imaging techniques: propagation-based imaging, analyzer-based imaging and grating interferometry," Opt. Express. 20(3), 2789 (2012).

[21] Pfeiffer, F., Kottler, C., Bunk, O., and David, C., "Hard X-ray phase tomography with low-brilliance sources," Phys. Rev. Lett. 98(10), 1-4 (2007).

[22] Szwaykowski, P. and Patorski, K., "Moire fringes by evolute gratings," Appl. Opt. 28(21), 4679 (1989).

[23] Pfeiffer, F., Bech, M., Bunk,, O., Kraft, P., Eikenberry E. F., Brönnimann C., Grünzweig C., and David C., "Hard-X-ray dark-field imaging using a grating interferometer.," Nat. Mater. 7(2), 134-137 (2008).

[24] Yang, F., Prade, F., Griffa, M., Jerjen, I.., Di Bella, C., Herzen, J., Sarapata, A., Pfeiffer, F., and Lura, P., "Dark-field X-ray imaging of unsaturated water transport in porous materials," Appl. Phys. Lett. 105(15), 154105 (2014).

[25] Prade, F., Fischer, K., and et al., "Time resolved X-ray Dark-Field Tomography Revealing Water Transport in a Fresh Cement Sample," Sci. Rep. 6, 29108 (2016).

[26] Pinzer, B. R., Cacquevel, M., Modregger, P., McDonald, S. A., Bensadoun, J. C., Thuering, T., Aebischer, P., and Stampanoni, M., "Imaging brain amyloid deposition using grating-based differential phase contrast tomography," Neuroimage. 61(4), 1336-1346 (2012).

[27] Olatinwo, M. B., Ham, K., McCarney, J., Marathe, S., Ge, J., Knapp, G., and Butler, L. G., “Analysis of Flame Retardancy in Polymer Blends by Synchrotron X-ray K-edge Tomography and Interferometric Phase Contrast Movies," J. Phys. Chem. B. 120(9), 2612-2624 (2016).

[28] Mohr, J., Grund, T., Kunka, D., Kenntner, J., Leuthold, J., Meiser, J., Schulz, J., and Walter, M., "High aspect ratio gratings for X-ray phase contrast imaging," in AIP Conf. Proc.1466(1), 41-50 (2012).

[29] Thüring, T., Abis, M., Wang, Z., David, C., and Stampanoni, M., "X-ray phase-contrast imaging at $100 \mathrm{keV}$ on a conventional source.," Sci. Rep. 4, 5198 ( 2014).

[30] Sarapata, A., Stayman, J. W., Finkenthal, M., Siewerdsen, J. H., Pfeiffer, F., and Stutman, D., "High energy x-ray phase contrast CT using glancing-angle grating interferometers.," Med. Phys. 41(2), 021904 (2014).

[31] Sarapata, A., Ruiz-Yaniz, M., Zanette, I., Rack, A., Pfeiffer, F., and Herzen, J., "Multi-contrast 3D X-ray imaging of porous and composite materials," Appl. Phys. Lett. 106 (15), 154102 (2015).

[32] P feiffer, F., Weitkamp, T., Bunk, O., and David, C., "Phase retrieval and differential phase-contrast imaging with lowbrilliance X-ray sources," Nat. Phys. 2(4), 258-261 (2006).

[33] Weitkamp, T., Diaz, A., Nohammer, B., Pfeiffer, F., Rohbeck, T., Cloetens, P., Stampanoni M., and David C., "Hard x-ray phase imaging and tomography with a grating interferometer," in Proc. of SPIE. 5535, 137-142 (2004).

[34] Schindelin, J., Arganda-Carreras, I., Frise, E., Kaynig, V., Longair, M., Pietzsch, T., Preibisch, S., Rueden, C., Saalfeld, S., Schmid, B., Tinevez, J.-Y., White, D. J., Hartenstein, V., Eliceiri, K., Tomancak, P., and Cardona, A., "Fiji: an open-source 
platform for biological-image analysis.," Nat. Methods. 9 (7), 676-682(2012).

[35] Perona, P. and Malik, J., "Scale-space and edge detection using anisotropic diffusion," IEEE Trans. Pattern Anal. Mach. Intell. 12 (7), 629-639 (1990).

[36] Münch, B.,"Xlib," ImageJ Wiki, <http://wiki.imagej.net/Xlib> (June 2015).

[37] Diamond, S., "A discussion of paper 'Patch microstructure in cement-based materials: Fact or artefact?' by Wong, H.S. and Buenfeld, N.R.," Cem. Concr. Res. 36(5), 998-1001 (2006).

[38] Otsu, N., "A Threshold Selection Method from Gray-Level Histograms,” IEEE Trans. Syst. Man. Cybern. 9(1), 62-66 (1979).

[39] Garcia, D., "OTSU," BioméCardio, <http://www.biomecardio.com/matlab/otsu.html>.

[40] Münch, B., and Holzer, L., "Contradicting geometrical concepts in pore size analysis attained with electron microscopy and mercury intrusion," J. Am. Ceram. Soc. 91(12), 4059-4067 (2008).

[41] Müller, B., Beckmann, F., Huser, M., Maspero, F., Székely, G., Ruffieux, K., Thurner, P., and Wintermantel, E., "Nondestructive three-dimensional evaluation of a polymer sponge by micro-tomography using synchrotron radiation," Biomol. Eng 19 (2-6), 73-78 (2002).

[42] Fierz, F. C., Beckmann, F., Huser, M., Irsen, S. H., Leukers, B., Witte, F., Degistirici, Ö., Andronache, A., Thie, M., and Müller, B., "The morphology of anisotropic 3D-printed hydroxyapatite scaffolds," Biomaterials, 29(28), 3799-3806 (2008).

[43] Banhart, J., [Advanced Tomographic Methods in Materials Research and Engineering], Oxford University Press, Oxford (2008).

[44] Chantler, C.T., Oslen, K., Dragoset, R.A., Chang, J., Kishore, A.R., Kotochigova,S.A., Zucker D.S.,"FFAST,” NIST, Physical Measurement Laboratory, <http://www.nist.gov/pml/data/ffast/index.cfm> (April 1997).

[45] Kaufmann, J., Loser, R., and Leemann, A., "Analysis of cement-bonded materials by multi-cycle mercury intrusion and nitrogen sorption,” J. Colloid Interface Sci. 336(2), 730-737 (2009).

[46] Moro, F. and Böhni, H., "Ink-bottle effect in mercury intrusion porosimetry of cement-based materials.," J. Colloid Interface Sci. 246, 135-149 (2002).

[47] Bennett, E. E., Kopace, R., Stein, A. F., and Wen, H., "A grating-based single-shot x-ray phase contrast and diffraction method for in vivo imaging," Med. Phys.37(11), 6047-6054 (2010).

[48] Hipp, A., Beckmann, F., Lytaev, P., Greving, I., Lottermoser, L., Dose, T., Kirchhof, R., Burmester, H., Schreyer, A., and Herzen, J., "Grating-based x-ray phase-contrast imaging at PETRA III," Proc.SPIE. 9212, 921206(2014). 